\title{
HIF-1 is the Commander of Gateways to Cancer Nagy MA*
}

Department of Chemistry, College of Science, Beni-Suef University, Beni-Suef, Egypt

\begin{abstract}
The hypoxia-inducible factor-1 (HIF-1) is primarily involved in the sensing and adapting of cells to changes in the $\mathrm{O} 2$ level, which is essential for their viability. An increased activity is recognized in the majority of clinical relevant hypoxic/ischemic episodes and human cancers . HIF-1 is considered a central regulator of the adaptation response of cancer cells to hypoxia that makes it a therapeutic target in solid tumors. In this article, the biochemical pathways that are regulated by HIF-1 and the factors that regulate HIF-1 expression are briefly discussed as targeting HIF-1, may selectively kill tumor cells that adapt to low $\mathrm{O} 2$ concentrations.
\end{abstract}

Keywords: Hypoxia; Hypoxia-inducible factor-1

\section{Introduction}

\section{Hypoxia definition}

Oxygen deprivation (hypoxia) occurs in tissues when $\mathrm{O} 2$ supply via the cardiovascular system fails to meet the demand of O2-consuming cells. Hypoxia occurs naturally in physiological settings (e.g. embryonic development and exercising muscle), as well as in pathophysiological conditions (e.g. myocardial infarction, inflammation, and solid tumor formation) [1].

Hypoxia, a common consequence of solid tumor growth in cancers, serves to propagate a cascade of molecular pathways which include angiogenesis that may be part of a self-regulated physiological protection mechanism preventing cell injury, especially under conditions of chronic ischemia [2].

Hypoxia is the main stimulus for angiogenesishence, hypoxic tumors

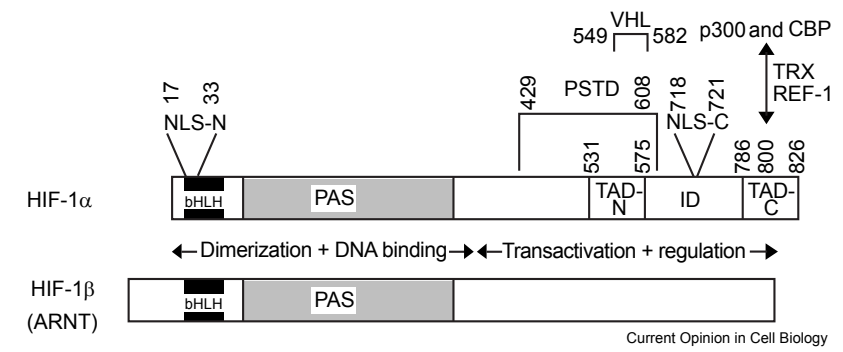

Figure 1: Structure and function of HIF-1. The HIF-1 $\alpha$ and HIF- $1 \beta$.

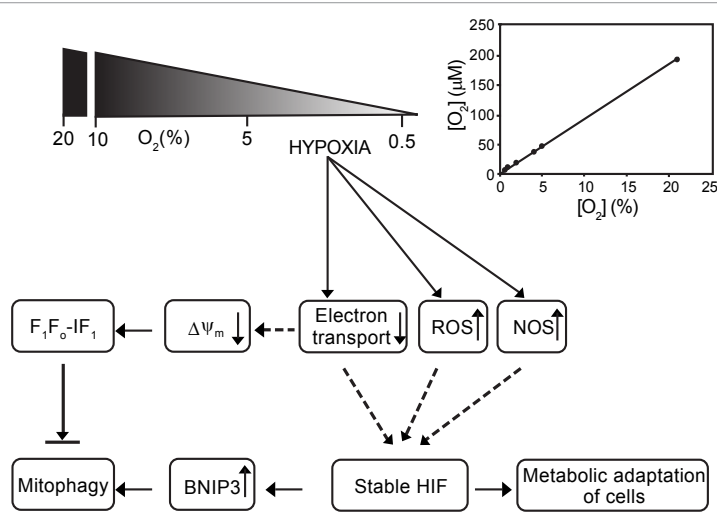

Figure 2: Major mitochondrial changes in hypoxia. are significantly more malignant, metastatic, radio and chemoresistant [3]. Hypoxia stimulates angiogenesis by signaling through Hypoxiainducible factors HIFs [4].

\section{Structure of HIF-1}

HIF- 1 is a heterodimer composed of HIF- $1 \alpha$ and HIF- $1 \beta$ subunits. Whereas HIF- $1 \beta$ is constitutively expressed, HIF- $1 \alpha$ expression is induced in hypoxic cells with an exponential increase in expression as cells are exposed to $\mathrm{O} 2$ concentrations of less than $6 \%$, which corresponds to a partial pressure (P) of $\mathrm{O} 2$ of approximately $40 \mathrm{~mm}$ $\mathrm{Hg}$ at sea level [5].

The amino-terminal half of HIF- $1 \alpha$ (amino acids 1-390) is necessary and sufficient for dimerization with HIF- $1 \beta$ and for DNA binding. HIF- $1 \alpha$ is ubiquitinated and subjected to proteasomal degradation in non-hypoxic cells Figure 1. Under hypoxic conditions, the fraction of HIF- $1 \alpha$ that is ubiquitinated decreases dramatically, resulting in an accumulation of the protein. A Pro-Ser-Thr rich protein stabilization domain is located between amino acids 429 and 608 of HIF-1a [6], subunits are shown with the basic helix-loop-helix (bHLH) and PERARNT-SIM (PAS) domains that are required for dimerization and DNA binding. Also shown for HIF-1 $\alpha$ are the amino-terminal $(\mathrm{N})$ and carboxyterminal (C) nuclear localization signal (NLS) and TAD; the Pro-Ser-Thr rich protein stabilization domain (PSTD; also known as the oxygen-dependent degradation domain); and sites of interaction with VHL, and p300 and CBP. The double-headed arrow indicates that reduction of Cys800, which is mediated by thioredoxin (TRX) and redox factor 1 (REF-1), is required for the interaction of TAD-C with cofactor p300 or CBP. The relevant amino acid residues are indicated numerically $[7,8]$.

\section{Stabilization of HIF-1}

Among recent advances are the discoveries that reactive nitrogen species (RNS) and oxygen species (ROS) participate in stability

*Corresponding author: Nagy MA, Department of Chemistry, College of Science, Beni-Suef University, Beni-Suef, Egypt, Tel: +20862375623, +20175011129; E-mail:nagy_bio@yahoo.com

Received October 02, 2010; Accepted November 13, 2010; Published Decembe 15,2010

Citation: Nagy MA (2011) HIF-1 is the Commander of Gateways to Cancer. J Cancer Sci Ther 3: 035-040. doi:10.4172/1948-5956.1000054

Copyright: @ 2011 Nagy MA. This is an open-access article distributed under the terms of the Creative Commons Attribution License, which permits unrestricted use, distribution, and reproduction in any medium, provided the original author and source are credited. 
regulation of HIF-1alpha and HIF-1 transactivation during normoxia Figure 2. Exposure of various cells to chemically diverse NO donors or conditions of endogenous NO formation under normoxic conditions induced HIF-1alpha accumulation, HIF-1-DNA binding, and activation of downstream target gene expression [9].

Hypoxia could decrease electron-transport rate determining $\Delta \psi \mathrm{m}$ reduction, increased ROS generation, and enhanced NO synthase. One (or more) of these factors likely contributes to HIF stabilization, that in turn induces metabolic adaptation of both hypoxic cells and mitophagy. Solid lines indicate well established hypoxic changes in cells, whilst dotted lines indicate changes not yet stated. Inset, relationships between extracellular $\mathrm{O} 2$ concentration and oxygen tension [10].

HIF-1 and metabolism of carbohydrates in hypoxia: When oxygen becomes limiting, cells reduce mitochondrial respiration and increase ATP production through anaerobic fermentation of glucose Figure 3. Also, hypoxia regulates almost all the enzymes involved in glycogen metabolism in a coordinated fashion, leading to its accumulation as a cellular response to hypoxia [11].

Tumors are exposed to intermittent hypoxia that could induce glycogen accumulation that could contribute to the resistance to fluctuations in blood supply that is commonly observed in tumors [12].

By stimulating the expression of glucose transporters and glycolytic enzymes, HIF-1 promotes glycolysis to generate increased levels of pyruvate. In addition, HIF-1 promotes pyruvate reduction to lactate

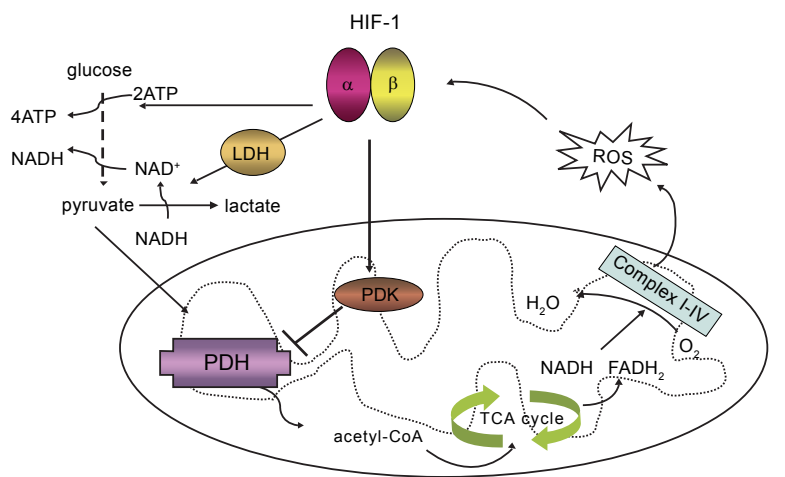

Figure 3: Multiple hypoxia-induced cellular metabolic changes are regulated by HIF-1.

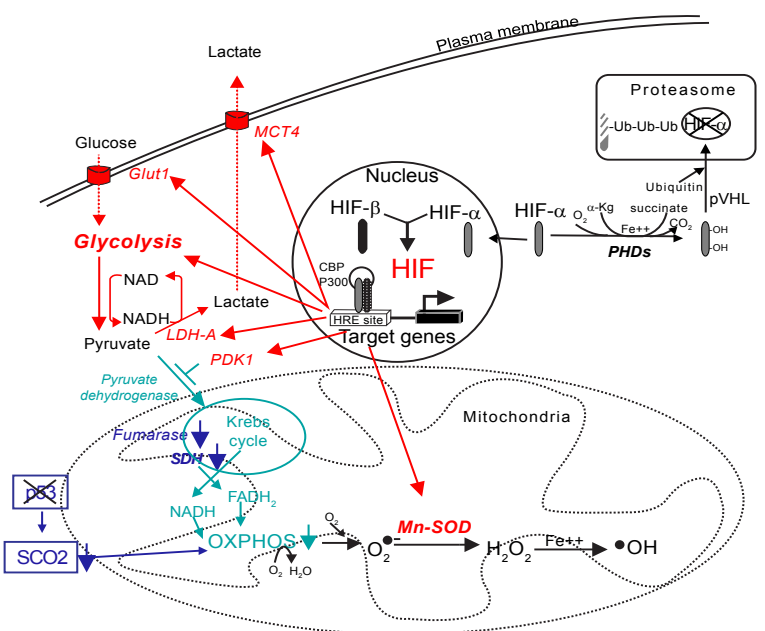

Figure 4: Glycolysis up-regulation and Oxidative phosphorylation.

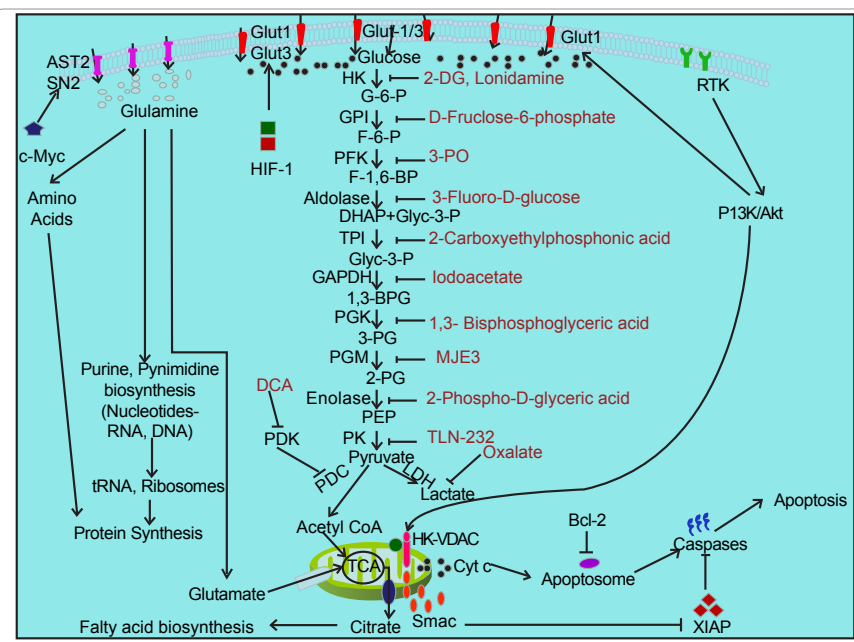

Figure 5: HIF-1 stabilization is one of opportunities in treating cancer by targeting cancer energy metabolism and mitochondria.

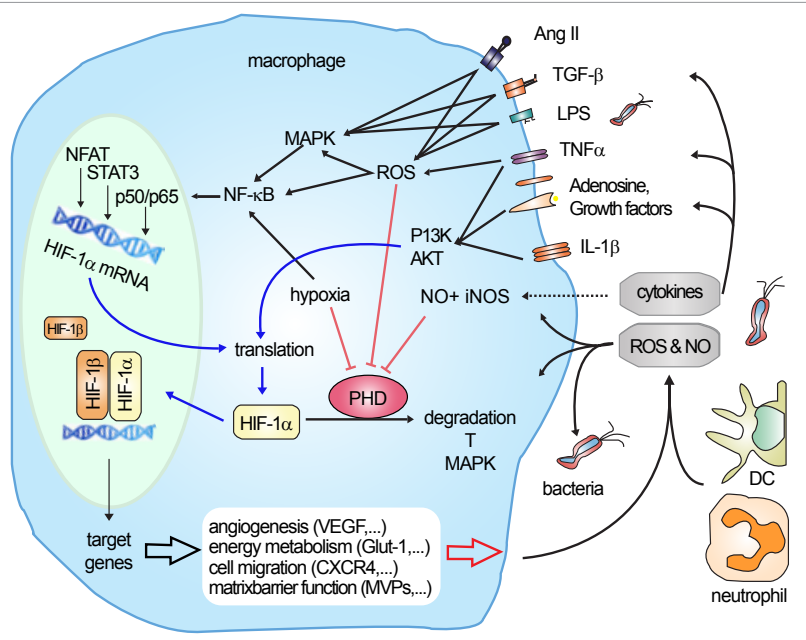

Figure 6: O2-(in) dependent regulation of HIF-1a in the inflammatory microenvironment.

by activating lactate dehydrogenase (LDH). Pyruvate reduction to lactate regenerates $\mathrm{NAD}+$, which permits continued glycolysis and ATP production by hypoxic cells. Furthermore, HIF-1 induces pyruvate dehydrogenase kinase 1 (PDK1), which inhibits pyruvate dehydrogenase and blocks conversion of pyruvate to acetyl CoA, resulting in decreased flux through the tricarboxylic acid (TCA) cycle.

Decreased TCA cycle activity results in attenuation of oxidative phosphorylation and excessive mitochondrial reactive oxygen species (ROS) production. Because hypoxic cells already exhibit increased ROS, which have been shown to promote HIF-1 accumulation, the induction of PDK1 prevents the persistence of potentially harmful ROS levels [13]. HIF-1alpha is necessary to support gluconeogenesis during liver regeneration that is necessary for hepatic glucose homeostasis during the reparative process [14].

HIF-1 and metabolism of carbohydrates in cancer cell: "waves" of gene expression that promote metabolic changes occur during carcinogenesis, beginning with oncogene-mediated changes, followed by hypoxia-induced factor (HIF)-mediated gene expression, both resulting in the highly glycolytic "Warburg" phenotype and suppression of mitochondrial biogenesis. The third (second oncogene) "wave" 
of adaptation stimulates glutaminolysis, that serves as an alternative pathway compensating for cellular ATP.

Together with anoxic glutaminolysis it provides pyruvate, lactate, and the NADPH pool (alternatively to pentose phosphate pathway). Retrograde signaling from revitalized mitochondria might constituent the fourth "wave" of gene reprogramming. thereby further promoting malignancy $[15,16]$.

In cancer cells, HIF-1alpha induces over-expression and increased activity of several glycolytic protein isoforms that differ from those found in non-malignant cells, including transporters (GLUT1, GLUT3) and enzymes (HKI, HKII, PFK-L, ALD-A, ALD-C, PGK1, ENO-alpha, PYK-M2, LDH-A, PFKFB-3). The enhanced tumor glycolytic flux triggered by HIF-1alpha also involves changes in the kinetic patterns of expressed isoforms of key glycolytic enzymes [17]. Some of the HIFlalpha-induced glycolytic isoforms also participate in survival pathways, including transcriptional activation of $\mathrm{H} 2 \mathrm{~B}$ histone (by LDH-A), inhibition of apoptosis (by HKII) and promotion of cell migration (by ENO-alpha) [18].

HIF-1alpha action may also modulate mitochondrial function and oxygen consumption by inactivating the pyruvate dehydrogenase complex in some tumor types Figure 4, or by modulating cytochrome c oxidase subunit 4 expression to increase oxidative phosphorylation in other cancer cell lines [19] (OXPHOS) down-regulation in cancers. Up-regulating HIF target genes (indicated in red) increases glucose entry, glycolytic flux, and conversion of pyruvate to lactate. In addition, it accelerates lactate exit from the cell. In parallel, PDK1-induced phosphorylation of pyruvate dehydrogenase reduces production of acetyl Coenzyme A. This diminishes substrate provision to the Krebs cycle and therefore contributes to decrease the flux through Oxidative phosphorylation.

Glucose (but not pyruvate) deprivation induced significant increase

Normoxia
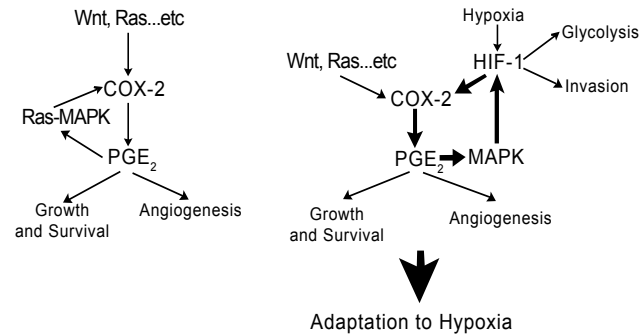

Figure 7: Model for COX-2 regulation and its role in colorectal tumorigenesis.

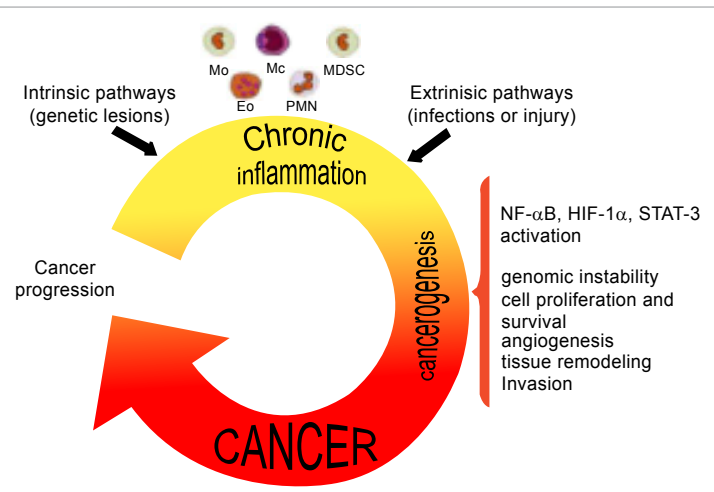

Figure 8: Inflammation and cancer connection. in VEGF transcription and secretion, but a rapid reduction in VEGFR2 protein synthesis and glycosylation, combined with a reduction in coreceptor neuropilin-1 (NRP-1) protein levels [20].

Moreover, Oxidative phosphorylation can produce ROS that can in turn attack mitochondrial proteins, lipids or DNA and thus further decrease Oxidative phosphorylation. Mutations in genes (indicated in blue) (fumarase, SDH) have been shown to be the cause of specific cancers. Invalidation of p53, very frequent in cancers, decreases the expression of SCO2, involved in cytochrome c oxidase assembly [21]. Cancer energy metabolism and mitochondria play a crucial role in tumor development. Increased glycolysis is a hallmark of most cancer cells. Various factors contribute to the phenomenon of the Warburg effect seen in tumors. Oncogenic alterations (PI3K/Akt) and HIF-1 stabilization result in increased expression of glucose transporters and glycolytic enzymes. Moreover, glycolysis aids in increasing the cellular anabolic processes by shunting intermediates to the pentose phosphate pathway [22], Figure 5.

HIF is a central station for cancer growth: Multiple signals affect transcription, translation or posttranslational modification of HIF-1a. These multiple signals, exemplified for macrophages, affect the protein amount of HIF-1 $\alpha$, the activity of HIF-1 and concomitant target gene expression [23], Figure 6.

Pathways blocking PHD activity are marked in red. Signaling pathways leading to an active HIF-1 $\beta /$ HIF- $1 \alpha$ heterodimer formation are given in blue $\rightarrow$ stimulation; $\dashv$ inhibition.

HIF-1is linker between inflammation and angiogenesis: Hypoxia promotes tumor progression by modulating gene expression. In colorectal tumor cells, COX-2 is transcriptionally induced by hypoxia via HIF-1, and it's up-regulation contributes to maintaining tumor survival and potentially promoting angiogenesis Figure 7. Thus, COX-2 overexpression can be regarded as a critical adaptive response to hypoxia, which mediates both short-(survival) and long-term adaptation (angiogenesis) [24].

Mutations in Wnt and Ras signaling pathways can induce COX-2 in normoxic a condition, resulting in increased levels of $\mathrm{PGE}_{2}$, which promotes tumor cell growth/survival and stimulates angiogenesis then activates the MAPK pathway and enhances HIF-1 transcriptional activity, resulting in a potential positive feedback loop that may act to maintain high COX-2 levels under hypoxic conditions [25]. In fastgrowing tumors, HIF-1alpha is involved in the activation of numerous cellular processes including resistance against apoptosis, overexpression of drug efflux membrane pumps, vascular remodeling and angiogenesis as it induces a number of genes integral to angiogenesis, e.g. Vascular endothelial growth factor (VEGF) [26] , Figure 8. VEGF can function on various types of cells, such as endothelial cells, hepatic stellate cells, endothelial progenitor cells and hemangiocytes, to induce vascular changes in HCC $[27,28]$. Endothelial cells (Ecs) proliferate in response to the hypoxia-induced VEGF and other growth factors secreted by the ECs or surrounding cell types, including hepatic stellate cells (HSC, considered liverresident pericytes), leukocytes, hepatocytes and Kupffer cells [29]. VEGF family members Increase vascular permeability; induce EC proliferation; leukocyte adhesion; regulate neovessel lumen diameter. Interaction of the VEGF with the receptor activates signaling pathways, e.g. PI3K/ Akt, Ras/Raf-MEK/ Erk, eNOS/NO, and IP3/Ca2+ [30]. VEGF-B specifically controlled endothelial uptake of fatty acids via transcriptional regulation of vascular fatty acid transport proteins that support the renergy requires for metastasis and development of cancer [31]. Irrespective of the trigger for the development both intrinsic (driven by genetic alteration) 


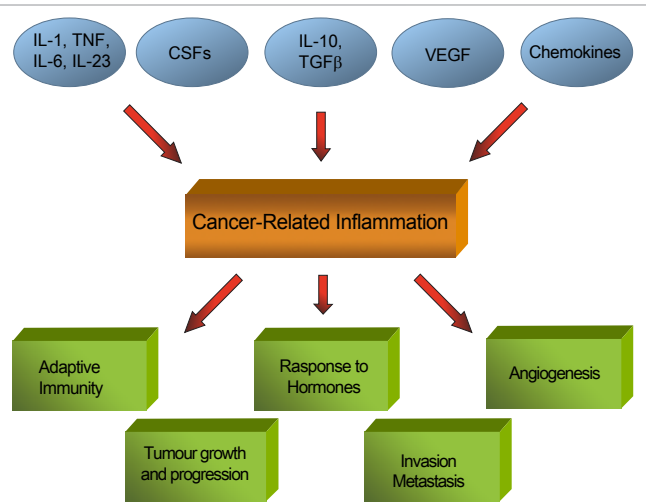

Figure 9: Cytokines, chemokines, and growth factors play the lead role in the crosstalk between tumor cells, blood vessel and infiltrating leukocytes.

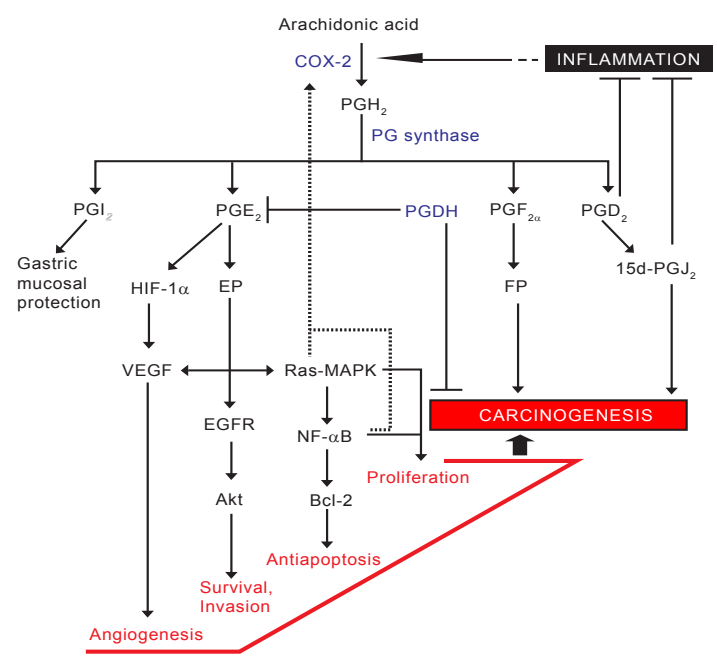

Figure 10: HIF-1 $\alpha$ is a bridge between inflammation and angiogenesis.

and extrinsic (driven by inflammatory cells and mediators) pathways result in inflammation and neoplasia. The transcription factors NF-kB, HIF-1a and STAT-3 are keymodulators of the inflammatory response that promotes cancer development [32], Figure 9. The presence of inflammatory components in the microenvironment of most neoplastic tissue most frequently results in enhanced angiogenesis, resistance to hormones (in hormonedependent tumours) and inhibition of adaptive anti-tumour immunity. Tumor cells survival, proliferation and eventually invasion and metastasis are all regulated by inflammatory mediators present at the tumor site [33], Figure 10.

The protein kinase D1 (PKD1), a newly described calcium/ calmodulin-dependent serine/threonine kinase, has been implicated in cell migration, proliferation and membrane trafficking. Increasing evidence suggests critical roles for PKD1-mediated signaling pathways in endothelial cells, particularly in the regulation of VEGF-induced angiogenesis [34], Figure 11. Phosphorylation is required for VEGFinduced microvessel sprouting in mouse aorta ring assay [35,36]. Angiogenesis plays a major role in chronic inflammation and may have prognostic value in disease progression [37]. The growth of new blood vessels is a dynamic yet highly regulated process that depends on coordinated signaling by growth factor and cell adhesion receptors [38].

\section{Genes induced by HIF-1 in cancer cells}

HIF-1 induces the genes of glucose transporters, enzymes of anaerobic glycolysis, factors and enzymes included in both angiogenesis and metastassis Figure 12.

ADM, adrenomedullin; ALDA, aldolase A; ALDC, aldolase C; AMF, autocrine motility factor; CATHD, cathepsin D; c-MET, hepatocyte growth-factor receptor 1; EG-VEGF, endocrine-glandderived VEGF; ENG, endoglin; ENO1, enolase 1; EPO, erythropoietin; ET1, endothelin-1; FN1, fibronectin 1; GLUT1, glucose transporter 1; GLUT3, glucose transporter 3; GAPDH, glyceraldehyde-3-phosphate dehydrogenase; HK1, hexokinase 1; HK2, hexokinase 2; IGF-2, insulin-like growth-factor 2; KRT14, keratin 14; KRT18, keratin 18; KRT19, keratin 19; LDHA, lactate dehydrogenase A; LEP, leptin; MMP2, matrix metalloproteinase 2; NOS2, nitric oxide synthase 2; TGFA, transforming growth-factor a; PFKL, phosphofructokinase L; PFKFB3, 6-phosphofructo-2-kinase/fructose-2,6-bisphosphatase 3; PKM, pyruvate kinase M; PGK1, phosphoglycerate kinase 1; TGF-b3, transforming growth-factor b3; TPI, triosephosphate isomerase; UPAR, urokinase plasminogen activator receptor; VEGFR2, VEGF receptor 2; VIM, vimentin [39].

\section{Regulation of HIF-1}

HIF-1a possesses bHLH and PAS domains which are involved in dimerization with HIF-1b and DNA binding. The HIF-1a subunit contains two TAD, the N- and the C- TADs. The N-TAD lies within the ODDD. The ODDD regulates the stability of HIF-1a via recognition, by the von Hippel-Lindau E3 ubiquitin ligase (pVHL), of the hydroxylation state of Pro402 and/or Pro564 residues. This hydroxylation is catalyzed by the enzyme PHD (prolyl hydroxylase domain protein). Acetylation

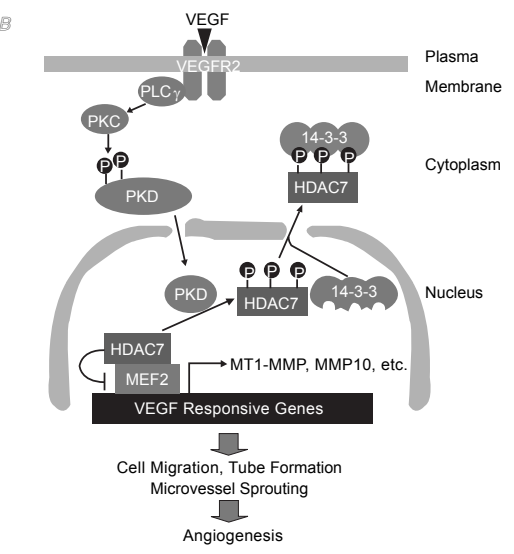

Figure 11: PKD1-dependent histone deacetylases 7HDAC7.

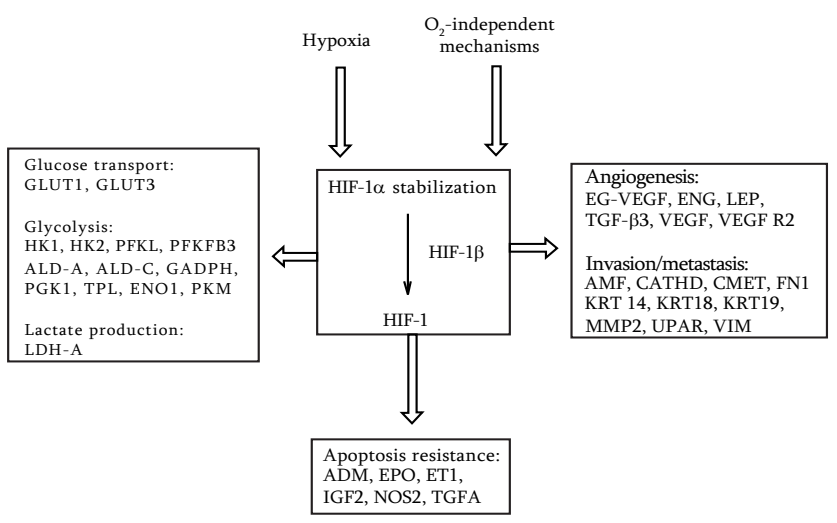

Figure 12: Genes induced by HIF-1 in cancer cells. 
of the Lys532 residue by the ARD1 acetyltransferase also favors interaction with pVHL. The hydroxylation state of the Asn 803 residue, by the enzyme FIH-1 (factor inhibiting HIF-1) inhibits binding of p300/ CBP, a HIF-1a co-activator. S-nitrosation of Cys800, in the C-TAD, also promotes HIF-1 transcriptional activity Figure 13. Three consensus sequences at Lys-391, -477 , and -532 may be modified by SUMO. The domain from 531 to 826 has been shown to be phosphorylated [40].

In the presence of oxygen HIFa undergoes prolyl hydroxylation at conserved residues, catalysed by a family of iron(II) $(\mathrm{Fe} 2+)$ and

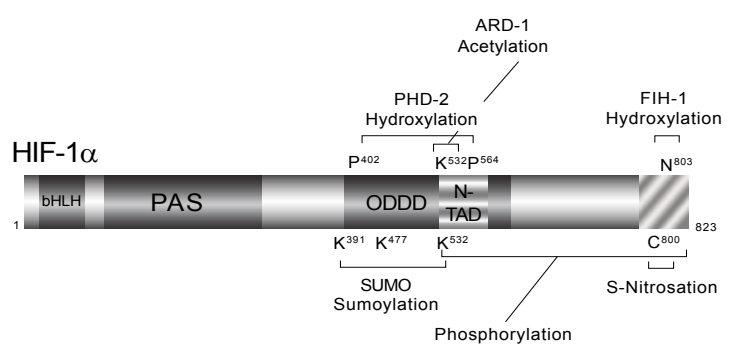

Figure 13: Domain structure of HIF-1a and targeted residues involved in its regulation.

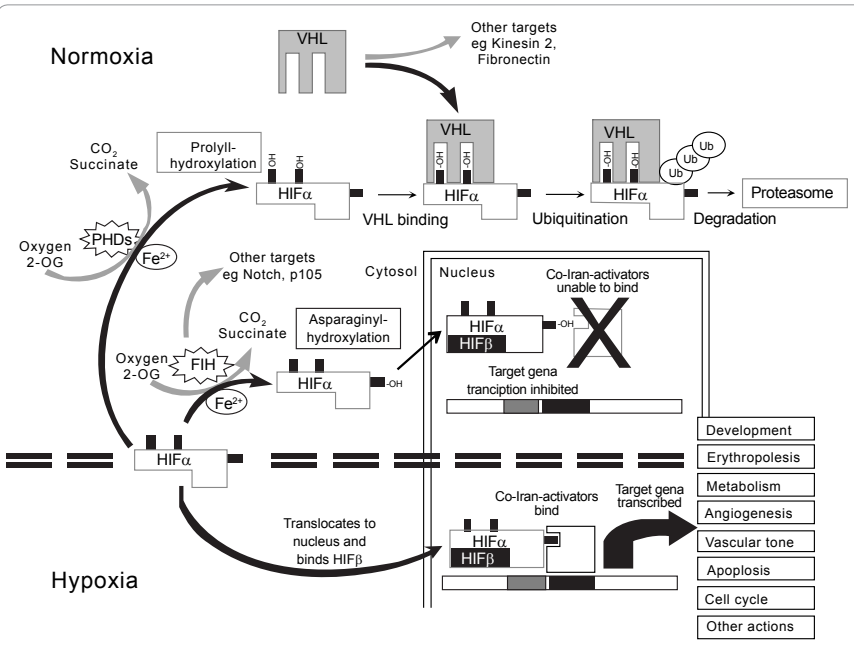

Figure 14: Regulation of Hypoxia Inducible Factor (HIF).

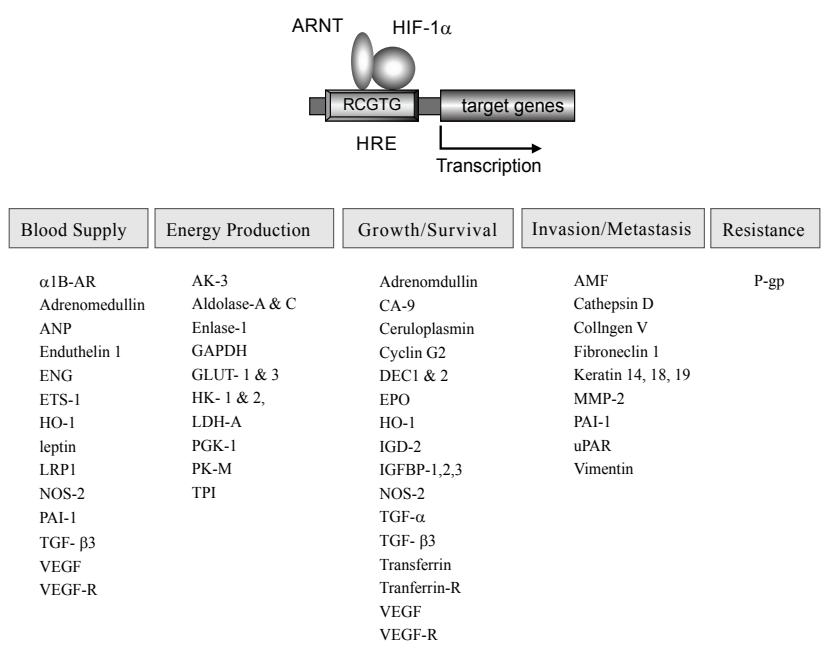

Figure 15: HIF-1-targeted genes related with tumor promotion.

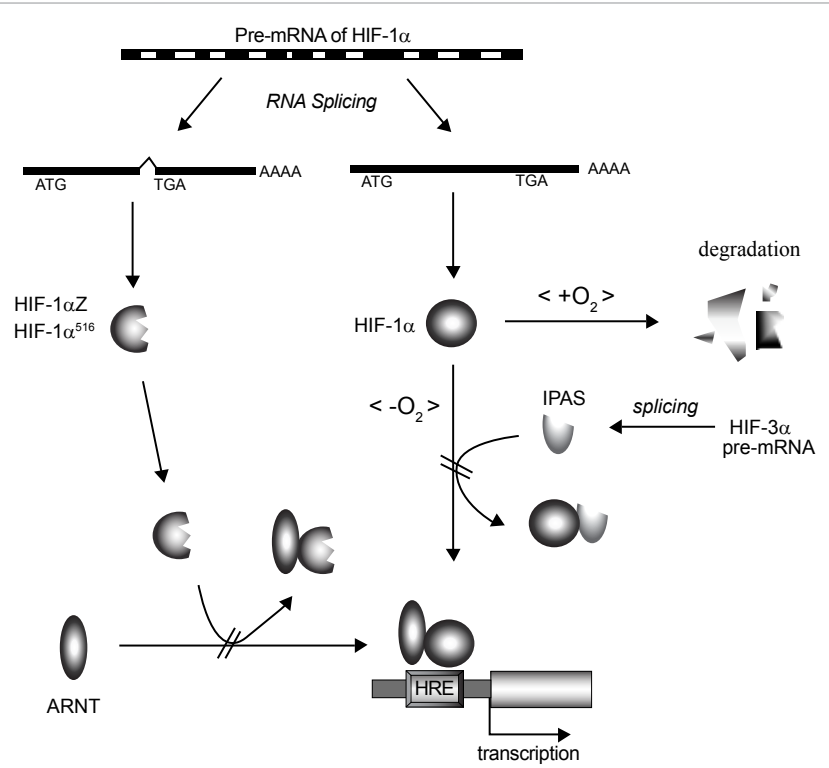

Figure 16: Inhibition of HIF-1 dimerization by HIF-1a and HIF-3a isoforms. Abbreviations: $\mathrm{O} 2$, normoxic conditions;_O2, hypoxic conditions.

2-oxoglutarate (2-OG) dependent prolyl hydroxylase domain (PHD) enzymes leading to recognition by the von Hippel-Lindau (VHL) protein which catalyses ubiquitination, targeting HIFa for proteasomal degradation. In addition, a conserved asparagine residue undergoes hydroxylation, by Factor Inhibiting HIF (FIH), which blocks activation of HIF target genes, as well as having other functions. In hypoxic conditions, HIFa translocates to the nucleus, binds to HIFa and additional co-transactivators to activate transcription of target genes which, in turn, modulate a wide variety of cellular processes [41], Figure 14. HIF-1-targete genes related to blood supply, energy production, growth, metastasis of cancer growth Figure 15.

Abbreviations: ADM, adrenomedullin; AK-3, adenylate kinase 3; AMF, autocrine motility factor; ANP, atrial natriuretic peptide; a1B$\mathrm{AR}$, a1B-adrenergic receptor; $\mathrm{CA}-9$, carbonic anhydrase 9; DEC1 and 2, differentiated embryo-chondrocyte expressed gene 1 and 2; ENG, endoglin; EPO, erythropoietin; GAPDH, glyceraldehyde-3-phosphate dehydrogenase; GLUT-1 and -3, glucose transporter 1 and 3; HK-1 and 2, hexokinase 1 and 2; HO-1, heme oxygenase 1; IGF-2, insulinlike growth factor 2; IGFBP-1, -2 and -3, IGF-binding protein 1, 2 and 3; LDH-A, lactate dehydrogenase A; LRP1, LDL-receptor-related protein 1; MMP-2, matrix metalloproteinase 2; NOS-2, nitric oxide synthase 2; P-gp, P-glycoprotein multidrug resistance transporter; PAI1, plasminogen activator inhibitor 1; PGK-1, phosphoglycerate kinase 1; PK-M, pyruvate kinase M; TGF-, transforming growth factor; TPI, triosphosphate isomerase; uPAR, urokinase plasminogen activator receptor; VEGF-R, VEGF receptor; VEGF [42]. Truncated PAScontaining proteins are translated from the alternative splicing of HIF1a or HIF-3a mRNA, and bind to the endogenous wild-types of HIF-1a or ARNT, thus competing with HIF-1a/ARNT dimerization. Hence, Inhibition of HIF-1 dimerization [42] Figure 16.

\section{Conclusion}

HIF-1a is the real play maker in the world of hypoxia, it is a cross link between carbohydrate metabolic cycles, inflammatory pathways and angiogenesis. It provides provided a therapeutic target and maybe even a clinical biochemical marker for diagnosis of cancer.

\section{Acknowledgements}

I appreciate the assistance and advice of Prof. Dr Bastawy M, College of 
Science, Beni Suef Univ. and Dr Kamal Adel Amin, College of Veterinary Medicine, Beni Suef Univ for kind co-operation.

\section{References}

1. Lou JJ, Chua YL, Chew EH, Gao J, Bushell M, et al. (2010) Inhibition of hypoxia-inducible factor-1alpha (HIF-1alpha) protein synthesis by DNA damage inducing agents. PLoS One 5: e10522.

2. Wu G, Luo J, Rana JS, Laham R, Sellke FW, et al. (2006) Involvement of COX-2 in VEGF-induced angiogenesis via P38 and JNK pathways in vascular endothelial cells. Cardiovasc Res 69: 512-519.

3. Airley RE, Mobasheri A (2007) Hypoxic regulation of glucose transport, anaerobic metabolism and angiogenesis in cancer: novel pathways and targets for anticancer therapeutics. Chemotherapy 53: 233-256.

4. Pugh CW, Ratcliffe PJ (2003) Regulation of angiogenesis by hypoxia: role of the HIF system. Nat Med 9: 677-684.

5. Jiang BH, Semenza GL, Bauer C, Marti HH (1996) Hypoxia-inducible factor 1 levels vary exponentially over a physiologically relevant range of $\mathrm{O} 2$ tension. Am J Physiol 271: c1172-c1180.

6. Salceda S, Caro J (1997) Hypoxia-inducible factor 1alpha (HIF-1alpha) protein is rapidly degraded by the ubiquitin-proteasome system under normoxic conditions. Its stabilization by hypoxia depends on redox-induced changes. J Biol Chem 272: 22642-22647.

7. Semenza GL (2001) HIF-1 and mechanisms of hypoxia sensing. Curr Opin Cell Biol 13: 167-171

8. Haase VH (2006) Hypoxia-inducible factors in the kidney. Am J Physiol Renal Physiol 291: F271-F281.

9. Brüne B, Zhou J (2003) The role of nitric oxide (NO) in stability regulation of hypoxia inducible factor-1alpha (HIF-1alpha). Curr Med Chem 10: 845-855.

10. Solaini G, Baracca A, Lenaz G, Sgarbi G (2010) Hypoxia and mitochondrial oxidative metabolism. Biochim Biophys Acta 1797: 1171-1177.

11. Pescador N, Villar D, Cifuentes D, Garcia-Rocha M, Ortiz-Barahona A, et al. (2010) Hypoxia promotes glycogen accumulation through hypoxia inducible factor (HIF)-mediated induction of glycogen synthase 1. PLoS One 5: e9644.

12. Cannito S, Novo E, Compagnone A, Valfrè di Bonzo L, Busletta C, et al. (2008) Redox mechanisms switch on hypoxia-dependent epithelial-mesenchymal transition in cancer cells. Carcinogenesis 29: 2267-2278.

13. Kim JW, Tchernyshyov I, Semenza GL, Dang CV (2006) HIF-1-mediated expression of pyruvate dehydrogenase kinase: a metabolic switch required for cellular adaptation to hypoxia. Cell Metab 3: 177-185

14. Tajima T, Goda N, Fujiki N, Hishiki T, Nishiyama Y, et al. (2009) HIF-1alpha is necessary to support gluconeogenesis during liver regeneration. Biochem Biophys Res Commun 387: 789-794.

15. Smolková K, Plecitá-Hlavatá L, Bellance N, Benard G, Rossignol R, et al. (2010) Waves of gene regulation suppress and then restore oxidative phosphorylation in cancer cells. Int J Biochem Cell Biol [Epub ahead of print]

16. DeClerck K, Elble RC (2010) The role of hypoxia and acidosis in promoting metastasis and resistance to chemotherapy. Front Biosci 15: 213-225.

17. Chen YQ, Zhao CL, Li W (2009) Effect of hypoxia-inducible factor-1alpha on transcription of survivin in non-small cell lung cancer. J Exp Clin Cancer Res 28:29.

18. Luo F, Liu X, Yan N, Li S, Cao G, et al. (2006) Hypoxia-inducible transcription factor-1alpha promotes hypoxia-induced A549 apoptosis via a mechanism that involves the glycolysis pathway. BMC Cancer 6: 26.

19. Marín-Hernández A, Gallardo-Pérez JC, Ralph SJ, Rodríguez-Enríquez S, Moreno-Sánchez R (2009) HIF-1alpha modulates energy metabolism in cancer cells by inducing over-expression of specific glycolytic isoforms. Mini Rev Med Chem 9: 1084-1101.

20. Adham SA, Coomber BL (2009) Glucose is a key regulator of VEGFR2/KDR in human epithelial ovarian carcinoma cells. Biochem Biophys Res Commun 390: 130-135.
21. Hervouet E, Simonnet H, Godinot C (2007) Mitochondria and reactive oxygen species in renal cancer. Biochimie 89: 1080-1088.

22. Pathania D, Millard M, Neamati N (2009) Opportunities in discovery and delivery of anticancer drugs targeting mitochondria and cancer cell metabolism. Adv Drug Deliv Rev 61: 1250-1275.

23. Nathalie Dehnea, Bernhard Brüne (2009) HIF-1 in the inflammatory microenvironment. Experimental Cell Research 315: 1791-1797.

24. Mas VR, Maluf DG, Archer KJ, Yanek KC, Fisher RA (2007) Angiogenesis soluble factors as hepatocellular carcinoma noninvasive markers for monitoring hepatitis $C$ virus cirrhotic patients awaiting liver transplantation. Transplantation 84: $1262-1271$.

25. Kaidi A, Qualtrough D, Williams AC, Paraskeva C (2006) Direct transcriptional up-regulation of cyclooxygenase-2 by hypoxia-inducible factor (HIF)-1 promotes colorectal tumor cell survival and enhances HIF-1 transcriptional activity during hypoxia. Cancer Res 66: 6683-6691.

26. Peng XH, Karna P, Cao Z, Jiang BH, Zhou M, et al. (2006) Cross-talk between epidermal growth factor receptor and hypoxia-inducible factor-1alpha signal pathways increases resistance to apoptosis by up-regulating survivin gene expression. J Biol Chem 281: 25903-25914.

27. Yang ZF, Poon RT (2008) Vascular changes in hepatocellular carcinoma. Anat Rec (Hoboken) 291: 721-734.

28. Pang R, Poon RT (2006) Angiogenesis and antiangiogenic therapy in hepatocellular carcinoma. Cancer Lett 242: 151-167.

29. Napoleone Ferrara, Hans-Peter Gerber, Jennifer LeCouter (2003) The biology of VEGF and its receptors. Nature Medicine 669-676.

30. Namiecińska M, Marciniak K, Nowak JZ (2005) [VEGF as an angiogenic neurotrophic, and neuroprotective factor]. Postepy Hig Med Dosw (Online) 59: 573-583.

31. Hagberg CE, Falkevall A, Wang X, Larsson E, Huusko J, et al. (2010) Vascular endothelial growth factor B controls endothelial fatty acid uptake. Nature 464 : 917-921.

32. Chiara Porta, Paola Larghi, Monica Rimoldi, Maria Grazia Totaro, Paola Allavena, et al. (2009) Cellular and molecular pathways linking inflammation and cancer. Immunobiology 214: 761-777.

33. Germano G, Allavena P, Mantovani A (2008) Cytokines as a key component of cancer-related inflammation. Cytokine 43: 374-379.

34. Ha CH, Jin ZG (2009) Protein kinase D1, a new molecular player in VEGF signaling and angiogenesis. Mol Cells 28: 1-5.

35. Chang Hoon Ha, Bong Sook Jhun, Hung-Ying Kao, Zheng-Gen Jin (2008) VEGF Stimulates HDAC7 Phosphorylation and Cytoplasmic Accumulation Modulating Matrix Metalloproteinase Expression and Angiogenesis. Arterioscler Thromb Vasc Biol 28: 1782.

36. Skurk C, Maatz H, Rocnik E, Bialik A, Force T, et al. (2005) Glycogen-Synthase Kinase3beta/beta-catenin axis promotes angiogenesis through activation of vascular endothelial growth factor signaling in endothelial cells. Circ Res 96 308-318.

37. Talaat RM (2010) Soluble angiogenesis factors in sera of Egyptian patients with hepatitis $C$ virus infection: correlation with disease severity. Viral Immunol 23: 151-157.

38. Stupack DG, Cheresh DA (2004) Integrins and angiogenesis. Curr Top Dev Biol 64: 207-238.

39. Manolescu B, Oprea E, Busu C, Cercasov C (2009) Natural compounds and the hypoxia-inducible factor (HIF) signalling pathway. Biochimie 91: 1347-1358.

40. Mazure NM, Brahimi-Horn MC, Berta MA, Benizri E, Bilton RL, et al. (2004) HIF1: master and commander of the hypoxic world. A pharmacological approach to its regulation by siRNAs. Biochem Pharmacol 68: 971-980.

41. Gale DP, Maxwell PH (2010) The role of HIF in immunity. Int J Biochem Cell Biol 42: 486-494.

42. Eun-Jin Yeoa, Yang-Sook Chunb, Jong-Wan Park (2004) New anticancer strategies targeting HIF-1. Biochemical Pharmacology 68: 1061-1069. 